\title{
\begin{tabular}{l|l} 
POLITIQUES \& & Politiques et management public
\end{tabular}
}

\section{La déontologie médicale face aux impératifs de}

marché

Medical deontology driven by market-based health care reform

Philippe Batifoulier, Franck Bessis et Olivier Biencourt

\section{OpenEdition}

\section{Journals}

Édition électronique

URL : http://journals.openedition.org/pmp/4139

ISSN : 2119-4831

\section{Éditeur}

Institut de Management Public (IDPM)

Édition imprimée

Date de publication : 15 mars 2011

ISSN : 0758-1726

\section{Référence électronique}

Philippe Batifoulier, Franck Bessis et Olivier Biencourt, «La déontologie médicale face aux impératifs de marché », Politiques et management public [En ligne], Vol 28/1 | 2011, mis en ligne le 02 octobre 2012, consulté le 10 décembre 2020. URL : http://journals.openedition.org/pmp/4139 


\title{
La déontologie médicale face aux impératifs de marché
}

\author{
Philippe Batifoulier ${ }^{*} a$, Franck Bessis ${ }^{b}$ et Olivier Biencourt ${ }^{c}$ \\ a EconomiX, Université Paris Ouest Nanterre La Défense, Philippe.Batifoulier@u-paris10.fr \\ ${ }^{b}$ Triangle, Université Lumière Lyon 2 ; fr.bessis@gmail.com \\ ‘GAINS, Université du Maine, Olivier.Biencourt@univ-lemans.fr
}

\section{Résumé}

Les médecins, au nom d'une déontologie qui leur serait propre, bénéficient du « privilège » de s'organiser en profession, c'est-à-dire comme un anti-marché. Déontologie et marché seraient donc supposés s'opposer. Dans cet article, nous développons une autre conception de la déontologie des médecins au moyen d'instruments d'analyse de l'économie des conventions et interrogeons les vertus supposées du marché. Nous montrons alors, les effets pervers de la pénétration de la mentalité marchande en santé. Le contexte normatif marchand, créé par la politique publique, influence la conception d'une activité médicale adéquate. Elle peut donc produire des effets non désirés : augmenter les dépenses et accroitre les problèmes d'accès aux soins.

(c) 2011 IDMP/Lavoisier SAS. Tous droits réservés

Mots clés : profession, déontologie médicale, économie des conventions, marchandisation, système de santé français.

\section{Abstract}

Medical deontology driven by market-based health care reform. Because of deontology, medical profession is an historical construction against market. In this paper, we develop an original conception of the medical deontology with the tools of the French school of the economics of conventions. We show how market reform in the French health sector modifies the definition of legitimate behaviour and impacts deontological rules. However, the development of a market-based normativity is the source of unexpected effects: more spendthrift behaviour and inequalities, contrary to the declared objective of health insurance governance.

(c) 2011 IDMP/Lavoisier SAS. Tous droits réservés

Keywords : profession, medical deontology, convention theory, marketisation, French health system, perversity effect.

*Auteur correspondant : Philippe.Batifoulier@u-paris10.fr

doi:10.3166/pmp.28.83-101 @ 2011 IDMP/Lavoisier SAS. Tous droits réservés 


\section{Introduction}

Règles déontologiques et logique marchande sont-elles compatibles? La déontologie participe à la justification du pouvoir d'autorégulation dont disposent les médecins (Hassenteufel, 1997; Robelet, 2005). La figure idéale du marché de concurrence pure et parfaite est de son côté le principal point d'appui normatif pour la critique d'un ordre professionnel, de ses barrières à l'entrée et du monopole dont il dispose (Skaked et Sutton, 1981). La cause semble donc entendue ; déontologie et marché sont deux modèles de coordination opposés et chacun correspond à une conception particulière du professionnel : tandis que, du point de vue de la déontologie, le médecin peut être appréhendé comme un responsable désintéressé, dans la perspective du marché il doit plutôt être conçu comme un entrepreneur opportuniste.

Bien que caricaturale, l'opposition précédente n'est pas automatiquement remise en cause par la prise en compte de défaillances de marché : les asymétries d'information entre médecins et patients ou entre médecins et tutelle sont analysées par l'économie orthodoxe à partir de modèles principal-agent. Ces derniers amplifient les dispositions calculatrices prêtées aux professionnels et guident l'élaboration de dispositifs incitatifs qui renforcent l'arbitrage marchand au détriment d'autres formes de coordination. Dans ces conditions, les a priori de l'économie orthodoxe sur la rationalité instrumentale des acteurs et l'efficacité de la coordination marchande préconisent de se débarrasser de l'illusion déontologique avec les eaux troubles de l'organisation professionnelle. Dans le domaine de la santé, cela va consister, dans l'intérêt des patients et pour l'équilibre des comptes de l'assurance-maladie, à chercher à piloter le plus possible les relations entre patients et médecins en amont de leur déroulement, aux moyens d'un système de rétributions et de sanctions supposé déterminer efficacement les comportements d'acteurs rationnels.

Ce cadre de pensée économique est l'un des éléments du contexte de domination idéologique du marché qui remet en question aujourd'hui l'autonomie et la qualité du travail professionnel dans plusieurs domaines comme la santé (Forest 2008, Chanial, 2010), la justice (Vauchez et Willemez, 2007 ; Favereau, 2010) ou encore la recherche (Bruno, 2008). La sociologie n'est toutefois pas en reste : succédant au fonctionnalisme de Parsons comme paradigme dominant, l'interactionnisme de l'école de Chicago s'est appliqué à nier toute spécificité, homogénéité et stabilité au travail des professionnels, réduisant les protections dont ceux-ci bénéficient au produit contingent de luttes (Champy, 2009). L'économie orthodoxe à partir d'un modèle de rationalité universel et la sociologie critique des professions à partir d'une posture relativiste ont ainsi en commun d' analyser indifféremment avocat, médecin et, pour reprendre un exemple de Howard Becker (2001), plombier. Conséquence de cette indifférenciation, la déontologie n'aurait rien à voir avec les conditions d'exercice particulières d'une activité professionnelle. Elle ne trouverait ni justification ni efficacité dans des caractéristiques propres aux services offerts. Cette déontologie relèverait avant tout d'une construction rhétorique au service du prestige et du contrôle des conditions d'accès à un marché.

Dans cet article, nous développons une autre conception de la déontologie des médecins au moyen d'instruments d'analyse de l'économie des conventions ${ }^{1}$. Il s'agit de comprendre

\footnotetext{
${ }^{1}$ Notamment, Salais et Thévenot (1986) ; Favereau (1988); Revue économique (1989) ; Batifoulier (2001) ; Orléan (2004) ; Eymard-Duvernay (2004 et 2006) ; et Diaz-Bone et Thévenot (2010) pour une explicitation des liens avec la sociologie pragmatique.
} 
à la fois les effets de la déontologie sur les comportements des médecins et, en amont, les effets d'une autre pression normative sur cette déontologie - celle de l'efficacité marchande, qu'elle soit supposée ou avérée (partie I). A partir de cette vision transformée des relations entre marché et déontologie, nous allons étayer la thèse selon laquelle la pénétration de l'idéologie marchande dans un secteur dominé par des valeurs non réductibles à l'intérêt privé, comme celui de la santé, produit la crise des organisations qu'elle vise à rationaliser. Depuis la fin des années soixante-dix, le système de santé et les institutions de la Sécurité Sociale ont fait l'objet d'une réévaluation comptable de plus en plus exclusive d'autres formes d'évaluation associées à d'autres objectifs de politique publique. Notre idée est que ce contexte normatif marchand créé par les politiques publiques influence la conception que se fait le médecin d'une activité adéquate, que ce soit par exemple en matière de durée de consultation ou de fixations des honoraires. Selon le schéma classique des «effets pervers $»^{2}$, ces politiques peuvent alors produire des effets contraires à ceux recherchés par le législateur en augmentant les dépenses et en accentuant les problèmes d'équité (partie II). Avant de développer ces deux points, nous allons dans le reste de cette introduction tirer avantage de rapprochements possibles entre la perspective conventionnaliste et d'autres travaux récents sur les professions pour préciser ce qui peut différencier constitutivement certaines professions.

Au regard de l'économie orthodoxe et de la sociologie critique, l'économie des conventions, propose une position intermédiaire qui se distingue par un «pluralisme sans relativisme » (Thévenot, 1992). Par rapport à l'orthodoxie économique, le pluralisme ouvre la théorie de l'action jusquelà cantonnée au calcul instrumental d'un individu prioritairement intéressé au gain monétaire à des formes de rationalité en valeur. Par rapport à l'interactionnisme, le refus de céder à un pur relativisme des valeurs consiste à prendre au sérieux les prétentions à l'universalité des acteurs (Boltanski et Thévenot, 1991) et des théories (Thévenot, 2006, Lemieux, 2009) en cherchant dans la longue durée et au sein de différents espaces sociaux les contraintes normatives les plus stables ${ }^{3}$. Cette position intermédiaire est nécessaire pour parler des qualités (Eymard-Duvernay, 1989 ; Favereau, Biencourt et Eymard-Duvernay, 2002) et plus particulièrement de la qualité du travail professionnel (Batifoulier et Gadreau, 2006 ; Bessis et Favereau, 2011). Ce faisant, les réflexions sur les professions menées à partir de cette approche peuvent dans une perspective socioéconomique apporter leur contribution au développement d'un « troisième regard » sur les professions auquel nous semblent participer en France des travaux comme ceux de Florent Champy (2011), Lucien Karpik (2007) ou Cyril Lemieux (2010) ${ }^{4}$.

Renvoyer parfaitement dos à dos les traditions orthodoxes en économie et interactionnistes ne serait cependant pas correct. Tandis que l'économie tente de faire disparaître toutes marges de manœuvre et se heurte au problème de l'incomplétude des règles, l'interactionnisme

\footnotetext{
${ }^{2}$ La thèse de l'effet pervers identifiée par Hirschman (1991) comme l'un des trois éléments principaux de la rhétorique réactionnaire est donc ici retournée contre les partisans du marché autorégulateur pour lesquels toute intervention de l'Etat dans les « lois » du marché serait contre-productive. Ce retournement tient simplement au fait qu'il apparaît clairement ici que l'Etat intervient dans la mise en place des marchés eux-mêmes.

${ }^{3}$ De manière synthétique, nous pouvons retenir que la culture professionnelle « évolue sous la contrainte de sa propre histoire » (Champy, 2011, p.142). La substance de chaque profession peut alors être objectivée sans perdre de vue l'idée qu'il s'agit de construction sociale (mais par là-même plus complètement arbitraire). ${ }^{4}$ Voir Champy (2009), pour la mise en perspective sociologique de ce « troisième regard » et la présentation d'autres travaux pouvant lui être associés.
} 
tire les enseignements de l'irréductible écart entre règles formelles et pratiques. Il s'agit même là d'un des fondements de l'indifférenciation défendue entre médecin et plombier. Chacun doit se référer à un ensemble de règles et mobiliser plus que ces règles pour les mettre en œuvre. C'est une idée sur laquelle insiste également l'économie des conventions qui semble donc pouvoir traiter aussi indifféremment plusieurs activités, comme celles du médecin et du plombier. Examinons ce point de plus près. Lorsqu' on raisonne sur l'économie orthodoxe à partir de l'économie des conventions, on peut le plus souvent tenir pour équivalentes trois propositions : ce sont les idées de rationalité limitée, d'incomplétude des règles et d'incertitude. Elles sont généralement confondues parce qu'elles ont toutes les mêmes conséquences principales : la remise en cause de la théorie du choix rationnel telle que modélisée par Savage (1954) et le rejet corrélatif de la figure traditionnelle de l'homo aconomicus. La justification de ce rejet est une première étape nécessaire pour justifier la construction alternative de l'homo conventionalis, quant à lui doté de capacités d'interprétation et de jugement (Bessis et al., 2006 ; Eymard-Duvernay, 2009). Cette autre théorie de l'action a une visée aussi générale que la théorie du choix rationnel. En cela, elle s'applique aussi bien au plombier qu'au médecin ou à l'avocat. A ce niveau demeure donc une forme d'indifférenciation, qui consiste non pas à déprécier les savoir-faire du médecin ou de l'avocat mais plutôt à réapprécier les compétences mobilisés pour d'autres métiers face à des situations incertaines ou des règles incomplètes (toutes les règles sont dites incomplètes, tous les acteurs sont supposés se prêter par moment à des activités d'interprétation, etc.). Franchissons maintenant une étape supplémentaire pour sortir de ce niveau d'indifférenciation générale.

Nous pouvons d'abord défendre a minima l'idée d'une différence de degrés (degrés d'incomplétude, d'incertitude, de complexité) entre les différents métiers. Cette idée encore trop vague suggère néanmoins déjà que c'est au sein de certaines professions comme celles de médecin ou d'avocat que l'on trouve l'archétype de l'homo conventionalis. Plus fondamentalement, pour retrouver ce qui différencie médecin et plombier à partir de la conception générale de l'action de l'économie des conventions, il faut entrer dans la distinction entre incomplétude et rationalité limitée : l'hypothèse d'incomplétude entraîne celle de rationalité limitée $e^{5}$ mais la réciproque n'est pas toujours vraie ${ }^{6}$. Cette distinction permet d'introduire la notion de « profession à pratique prudentielle » proposée par Champy (2011, p.151) : «Une façon d'exprimer la différence consiste à dire que les problèmes auxquels les plombiers sont confrontés peuvent toujours être décrits exhaustivement en termes concrets, même si cela n'est pas simple. A l'inverse, les professions à pratique prudentielle font face à des problèmes irréductibles à ce type de description. C'est évident pour ce qui touche au vivant : un médecin ne peut jamais être catégoriquement certain d'avoir pris en compte tout ce qui doit l'être pour soigner un malade, car il ne peut pas se livrer à une description exhaustive de ce dernier ». Cette différence empêche dans le second cas d'aboutir à des choix parfaitement indiscutable ${ }^{7}$. Elle appelle au contraire une délibération sur les finali-

\footnotetext{
${ }^{5}$ En l'absence d'un ensemble complet d'états du monde, l'agent ne peut pas optimiser. L'axiome de Savage (1954) qui postule un préordre complet sur les préférences n'est pas respecté.

${ }^{6}$ Les limites de nos capacités cognitives nous empêchent par exemple de dresser la liste exhaustive des stratégies possibles lors d'une partie d'échec mais cet ensemble de stratégie est bien complet, contrairement à la trajectoire d'évolution d'organismes vivants qui donne à penser un ensemble ouvert de possibilités. ${ }^{7}$ Qu'il s'agisse de mobiliser des règles de science médicale ou des règles déontologiques.
} 
tés de l'activité et requiert alors une certaine forme d'indépendance (ou « autonomie de réflexion »(Champy, 2011, p.158)), autorisée et en partie rendue possible par la déontologie et l'organisation en ordre professionnel (conçue ainsi comme un « dispositif de réflexivité » (Favereau, 2010, p.126) $)^{8}$.

Par la suite, nous allons considérer la tarification de la prestation médicale au sens large (comprenant actes gratuits, dépassement d'honoraires, renvois vers des confrères, etc.) comme une composante de l'activité prudentielle désorganisée par la mise en place de dispositifs incitatifs. Ces dispositifs visent un contrôle mécanique en privilégiant la rationalité instrumentale sur la dimension prudentielle de l'activité. Ils tendent à déposséder les professionnels du sens de leur activité et à réduire ainsi cette dernière à une simple source de revenu monétaire. Cette opération correspond à un programme de politique économique. Le marché a besoin d'individus conformes à ses impératifs. Toutefois, les dispositifs d'incitation ne pouvant pas faire complètement disparaître les marges d'autonomie, celles-ci se trouvent de plus en plus systématiquement exploitées par des individus réduits à l'opportunisme.

\section{La promotion du marché contre la déontologie professionnelle}

Le caractère vague et général de la déontologie entretient le pouvoir discrétionnaire du médecin. Certes le code de déontologie médicale proscrit la discrimination dans l'accès aux soins, la publicité, la compétition, etc. Mais il le fait au moyen de formules abstraites comme « la médecine ne doit pas être pratiquée comme un commerce » (article 19), ou encore « les médecins doivent entretenir entre eux des rapports de bonne confraternité » (article 56). On peut donc douter que la déontologie soit réellement prescriptive car elle offre de larges espaces de liberté aux médecins. Le texte existe bien et se trouve parfois brandi par la profession, mais rien ne garantie qu'il se traduise en action bienveillante. C'est particulièrement manifeste pour les articles destinés à codifier les rapports entre médecins et patients (Mossé, 2005). Ainsi, en matière de durée de consultation, « il faut élaborer un diagnostic avec le plus grand soin, en y consacrant le temps nécessaire» (article 33). De même pour le montant des honoraires qui «doivent être déterminés avec tact et mesure » (article 53). Le serment d'Hippocrate renforce d'ailleurs ce constat, par exemple lorsqu'il fait jurer au nouveau médecin d'être « fidèle aux lois de l'honneur et de la probité ». A partir d'une déontologie aussi évasive en matière de relation médecin-patient, comment prétendre la laisser régir le colloque singulier?

En considérant l'incomplétude des textes comme point final de l'analyse la déontologie ne semble être qu'un habillage astucieux pour permettre à la profession de négocier avec l'Etat des droits d'autorégulation et d'autocontrôle. Il est donc tentant de condamner la déontologie au regard de son incomplétude - c'est la position de l'économie de la santé moderne (Bloche, 2001). L'économie des conventions fait pour sa part de l'incomplétude des règles un point de départ de l'analyse. Toutes les règles sont incomplètes, tous les acteurs sont supposés compléter la règle lors d'un exercice d'interprétation qui revient à donner un sens à la règle et, par là, orienter l'action. Interpréter la règle revient à arbitrer

\footnotetext{
${ }^{8}$ L'exemple des journalistes, où l'autonomie apparaît aussi comme une « nécessité de la pratique » (Lemieux, 2010, p.25) montre que d'autres formes d'organisation peuvent se substituer à l'ordre professionnel, qui appellent dans tous les cas un renforcement des dispositifs démocratiques (Lemieux, 2010b, p.128).
} 
entre une pluralité de principes acceptables et à donner ainsi un sens particulier à cette règle.

Dans cette nouvelle perspective, nous pouvons rendre visible une autre manière pour l'Etat de s'opposer à la profession médicale. Par différentes formes d'action et de communication, l'Etat rend plus naturel, à la fois sur un plan symbolique et matériel, le recours à un principe d'interprétation particulier ${ }^{9}$. Autrement dit, il peut orienter l'interprétation en cours d'action à laquelle se livrent les acteurs. Si le principe supérieur marchand est celui qui vient donner sens à la règle déontologique incomplète, il devient possible de rapprocher le fonctionnement d'une profession de celui d'un de marché, de manière à soumettre cette profession médicale aux impératifs économiques fixés par la tutelle. La promotion du marché par l'Etat est alors une façon de combattre le pouvoir monopolistique de la médecine. C'est l'Etat qui va porter la pression idéologique du marché dans le but d'enrayer le pouvoir médical.

Cette marchandisation est d'une autre nature que celle qui existe au travers du rôle important des organisations marchandes qui animent le secteur de la santé, qu'il s'agisse de l'industrie pharmaceutique, des établissements de soins à but lucratif ou des organismes d'assurance complémentaire. En ciblant le médecin, la promotion du marché n'a plus besoin de frontière ou de lieu (Favereau, 2010b). Le médecin, par un jeu d'incitation, est invité à adopter une logique marchande, quelque soit son lieu d'exercice : cabinet de ville ou hôpital public. Cette entreprise est audacieuse car le médecin se considère, notamment en raison de sa déontologie, comme un rempart à la dénaturation marchande des activités de santé. Mais c'est précisément à partir de l'incomplétude de la déontologie que la posture marchande du médecin est construite. Il suffit de capitaliser ses attributs d'homoeconomicus et de s'appuyer sur l'expression d'un intérêt privé que l'on reconnaît derrière l'exercice d'un pouvoir discrétionnaire ou l'instrumentalisation d'un différentiel d'information. En d'autres termes, la formalisation du médecin comme individu rationnel cherchant à satisfaire son intérêt individuel n'est plus seulement une hypothèse théorique mais doit être un idéal à atteindre pour la politique économique. Dans cette perspective, si l'on reconnaît que le médecin peut manifester des attitudes bienveillantes vis-à-vis du patient, celles ci sont associées à un altruisme instrumental. La déontologie professionnelle n'est qu'un instrument de fidélisation d'une patientelle ou de développement d'une réputation. Cette conception réduit toutes les valeurs professionnelles à l'intérêt égocentrique et cherche à contester tout ce qui fait l'identité collective d'une profession.

Cette requalification du médecin vise à le désencastrer de la profession médicale pour le réencastrer dans un ordre marchand et l'inciter à adopter une posture adéquate au registre concurrentiel. Il convient alors de casser le collectif en en appelant à la rationalité individuelle opportuniste. La réalisation de ce programme marchand peut prendre différentes formes. En matière de santé, le marché pénètre le secteur par l'intermédiaire de la notion de concurrence, considérée comme une pragmatique nécessaire à la maîtrise des coûts (Rochaix, 2004) et par la diffusion d'un comportement entrepreneurial, nécessaire à l'activation de la logique de compétition (Saltman, 2002). A l'hôpital, la mise en avant de la chasse aux coûts et de la nocivité des déficits ont conduit à relativiser la spécificité

${ }^{9}$ L'Etat peut donc faciliter ou au contraire restreindre l'accès à certains mondes de production et principes supérieurs communs. Voir Salais et Storper (1995), partie IV, chapitre 1, pour une présentation plus détaillée (et moins schématique !) de cette idée. 
de la gestion publique pour considérer que les règles de bonne gestion viennent du privé. L'orientation concurrentielle s'instruit autour du calcul d'un prix via les points ISA (dans le cadre de la T2A), à partir de la comptabilité analytique, et peut conduire à activer une logique de compétition (Moisdon, 2000). Cette concurrence «encadrée », « fictive » ou «par comparaison » adapte la solution marchande aux spécificités hospitalières (Mossé et Pierru, 2002 ; Domin, 2008). Elle fait émerger un pouvoir gestionnaire et une culture du management pour corriger le pouvoir médical en développant une logique contractuelle et l'institution d'une rémunération à la performance. En médecine de ville, l'institution d'un marché est plus difficile. Les individus (offreurs comme demandeurs de soins) ne se comportent pas spontanément comme des preneurs de prix et le prix des soins n'est traditionnellement pas (ou peu) un argument de la décision de soins. L'idée concurrentielle va alors transiter par le patient invité à mobiliser de nouvelles compétences et de nouveaux droits. Il est dorénavant appeler à faire jouer la concurrence et à exercer une contrainte de marché (Batifoulier et al., 2008 ; Domin, 2010, Laude, 2005). Les agences d'Etat, productrices d'une information labellisée et les intermédiaires de la qualité vont « coacher » le patient pour le faire accéder à un statut de consommateur responsable. Le médecin est alors invité à amender son paternalisme historique pour se caler dans les valeurs d'autodétermination, de non ingérence et de gouvernement de soi qui développent une confiance plus consumériste.

Cette «marchandisation» se traduit par un engagement qualitatif de l'État quand il cherche à exporter les valeurs du marché dans un secteur dit «à imperfections » comme la santé. La concurrence, qui est une contre valeur pour la déontologie médicale, va être mobilisée par le régulateur pour contrecarrer le pouvoir du médecin. Dans ces conditions, c'est bien la concurrence en tant que valeur normative, davantage que les techniques concurrentielles d'un marché pur et parfait, qui vont être mobilisées par le législateur.

Ce déplacement normatif sur lequel se construit le marché de la santé repose sur un comportement considéré comme universel : la recherche de l'intérêt privé. Dans le cas des médecins, la déontologie n'est qu'un véhicule spécifique de ce comportement.

La construction d'un marché ne vise pas ainsi à combattre l'opportunisme des médecins car celui-ci est considéré comme indépassable. Elle vise, par un mécanisme d'incitations bien choisi, à orienter cet opportunisme vers des attitudes valorisées par la tutelle parce qu'elles sont économes en dépenses de santé (Batifoulier et al., 2007). Les individus n'adoptent pas spontanément un comportement de marché. En matière de santé,l'absence de prix de marché ne permet pas d'harmoniser les intentions individuelles et de totaliser les offres et les demandes. Il faut donc reconstruire un système de prix pour construire un marché. Le régulateur central va donc fixer des prix paramétriques pour stimuler un marché. Il va reproduire fictivement un fonctionnement de marché en développant un système de règles dont l'objectif est d'envoyer des signaux vers les producteurs et les consommateurs (Grignon, 2006).

Du fait même de leur visée, ces règles ne peuvent être des règles de contrôle. Elles ont pour objectif de modifier ex ante (et non ex post) les comportements des acteurs. Dans cette perspective, l'incitation est préférée à la coercition. Alors que sur un marché classique, il convient d'identifier les bons prix, sur ce marché fictif, il faut trouver les bonnes règles, celles qui vont inciter les individus à adopter un comportement conforme aux souhaits du régulateur central. La politique économique a alors pour mission de développer des incitations qui vont envoyer des signaux en direction des acteurs de santé, les engageant à adopter des comportements « optimaux ». 
Cette logique a pénétré le droit de l'assurance maladie dont l'étude montre que les économies budgétaires ont été recherchées dans le développement d'une logique incitative visant à contrecarrer l'opportunisme supposé des acteurs (Vacarie et al., 2008 ,). Les choix de gouvernance de l'assurance maladie s'appuient sur des individus supposés rationnels et intéressés, qui ont alors moins affaire à des règles contraintes. Ils sont plutôt mis en mesure de calculer les gains ou les pertes de différentes opportunités (bonus/malus) : se conformer au parcours de soins, consommer du médicament à « service médical rendu faible », prescrire des médicaments génériques, respecter les protocoles standardisés, etc.

L'étude empirique des conventions médicales ${ }^{10}$ et en particulier de moments clefs comme l'institution d'un double secteur tarifaire ou, à un degré moindre, l'existence de conventions séparées entre généralistes et spécialistes, vient également nourrir l'hypothèse d'expansion du régime marchand. Une analyse lexicale de ces conventions confirme le développement d'une normativité marchande qui vient concurrencer les autres logiques (Abecassis et Domin, 2009). On peut ainsi mettre en évidence une évolution progressive du langage et des représentations qu'il implique : d'un langage principalement axé sur l'efficacité de l'organisation administrative du système de santé, on a lentement glissé vers une représentation comptable et un effort centré avant tout sur la maîtrise des dépenses.

Ce mouvement du droit de l'assurance maladie est essentiel si l'on met en avant sa fonction heuristique. Le droit est un instrument primordial de justification du comportement des acteurs. La règle de droit fournit une référence normative qui lui confère une fonction «éducative ». "Cette fonction éducative de la loi qui vise à instaurer une hiérarchie dans les valeurs et à en préconiser certaines est aussi importante que ses fonctions de dissuasion et de répression » (Hirschman, 1985, p. 94). Qu'elle soit justifiée, contestée, effective ou non, la règle fournit dans tous les cas un repère pour l'action. Cette influence de la règle de droit sur les représentations a des conséquences importantes sur l'efficacité des dispositifs incitatifs. Les prévisions en la matière (qui justifient l'usage de ces dispositifs) sont élaborées en supposant que les préférences des agents restent inchangées (seuls changeraient les comportements) tandis que ces politiques ont pour premier effet d'orienter d'un point de vue cognitif (avant d'inciter d'un point de vue plus utilitariste) les agents vers l'adoption d'un autre système de préférences (d'autres représentations et d'autres formes de jugements). L'évolution marchande du droit de l'assurance maladie est alors de nature à déplacer la conception d'un comportement légitime.

Dans cette construction marchande par l'incitation, une déontologie qui en appellerait à d'autres valeurs que le self interest ne serait pas seulement inutile. Elle serait encombrante car elle pourrait avoir un effet contreproductif sur les ambitions de la tutelle.

Considérer que la déontologie puisse être le support d'une autre gamme de comportement plus proche d'une éthique (au sens commun du mot) peut modifier cette conception. Dans cette éventualité, la marchandisation édifie des valeurs qui vont se confronter à d'autres valeurs. Ce télescopage de valeurs peut alors produire des effets non souhaités.

\footnotetext{
${ }^{10}$ La technique de la convention médicale, à l'image d'une convention collective, habilite les syndicats de médecins et les caisses de Sécurité sociales à négocier les tarifs des soins. Ce tarif s'impose aux médecins et sert de base aux remboursements des caisses aux assurés sociaux.
} 


\section{De nouveaux fondements normatifs à la rationalité médicale. Les effets pervers de l'orientation marchande}

L'orientation marchande du secteur de la santé prend place dans un contexte où sont déjà présentes d'autres valeurs. La déontologie médicale peut en effet également être associée à l'expression de valeurs hippocratiques et libérales, caractéristiques de la manière dont « pense l'institution médicale » (Jaunait, 2005).

Les valeurs hippocratiques s'appuient sur le respect de l'autonomie du patient qui doit participer au processus thérapeutique, sur la «non malveillance » (il s'agit de ne pas nuire au patient, « primum non nocere ») et sur la bienveillance qui vise à apporter un bénéfice net au patient. Associées à ces valeurs, l'existence d'une forme de justice distributive oblige à délivrer des soins de manière juste et appropriée, en appréciant le besoin du patient (Gillon, 1994).

Les valeurs libérales sont quant à elle sédimentées dans la " charte de la médecine libérale », votée en 1928 et dont le code de la Sécurité Sociale reprend certains aspects. Ses principes sont conçus comme autant de remparts aux menaces de «salarisation » voire de «fonctionnarisation » que ferait peser la politique publique sur le corps médical : en particulier, le principe de «l'entente directe » entre le patient et le médecin interdit toute remise en cause du paiement à l'acte ; le libre choix du médecin par le patient condamne l'affiliation d'un patient à un médecin (et le paiement de ce dernier par capitation); la liberté d'installation n'est pas compatible avec des politiques luttant contre les déserts médicaux, etc.

Les médecins sont donc animés par différentes conceptions de la pratique médicale légitime et le compromis entre ces valeurs est également différent selon les segments médicaux et leur lieu d'exercice. Cette pluralité est aujourd'hui remise en cause par la logique marchande promue par l'Etat. Cette dernière est plus en phase avec les valeurs libérales, qu'elle renforce alors au détriment des valeurs hippocratiques. Notre thèse est qu'en défaisant de la sorte tous les compromis antérieurs, la politique de marchandisation s'accompagne d'effets pervers dont nous allons maintenant présenter la logique.

Pour souligner les tensions entre différentes valeurs, différents ordres de motivation ou différents systèmes de préférences, nous allons nous appuyer sur des résultats de psychologie sociale et cognitive (Déci, 1971) qui ont déjà fait l'objet d'importations dans la théorie économique (Frey 1997, Kreps, 1997). Spécifiant à leur façon la distinction entre rationalité axiologique et instrumentale, les psychologues sociaux déclinent les motivations en motivations intrinsèques et extrinsèques. Les motivations intrinsèques (MI) concernent la satisfaction directe que l'on peut tirer d'une activité, sans en attendre de récompense ni chercher à éviter un sentiment de culpabilité ; elles sont sources de satisfaction en soi. Les motivations extrinsèques $(\mathrm{ME})$ concernent les récompenses exogènes à l'activité qu'elles soient matérielles (rémunération monétaire) ou symboliques (approbation de l'entourage) dans une perspective intéressée et conséquentialiste. Cette distinction permet de mettre au jour et de tester empiriquement les effets pervers de dispositifs visant à stimuler les ME lorsque ceux-ci ont un effet inhibiteur voire destructeur sur les MI $^{11}$. L'expérience de

\footnotetext{
11 Voir Bénabou et Tirole (2003) pour la présentation modélisée d'un argument similaire, mais dans le cadre d'une relation principal agent, qui génère alors une tension entre les hypothèses de rationalité retenues et le principe même de l'argument : parler de motivation intrinsèque ne revient-il pas à sortir du cadre de l'action instrumentale modélisée par la théorie du choix rationnel (Da Silva, 2011)?
} 
Gneezy et Rustichini (2000) sur les gardes d'enfant est particulièrement révélatrice ${ }^{12}$. Cette expérience se déroule sur 20 semaines. A la quatrième, on introduit une amende quand les parents viennent chercher leur enfant en retard à la garderie, à la dix-septième, on la supprime. Un « groupe de contrôle» est constitué sans amende. Pour le groupe test, le nombre de retards augmente avec l'instauration de l'amende puis se stabilise à un niveau élevé (plus important que celui du groupe de contrôle). La suppression de l'amende ne change rien. Les auteurs en déduisent une destruction durable des MI : l'amende a, à la fois, légitimé le retard (on paye pour être en retard) et détruit l'adhésion à une norme sociale.

La possibilité d'un effet d'éviction ( « crowding out effect ») où l'existence de ME vient détruire ou altérer les MI compromet l'efficacité des incitations financières. En effet, pour la théorie de l'agence, si les MI existent, elles vont dans le même sens que les ME et accélèrent la performance de l'individu (effet « crowding in »). Il en va autrement si les motivations sont substituables et non complémentaires. Dans ce cas, ce n'est pas seulement l'hypothèse de désutilité de l'effort qui est remise en question, c'est aussi la performance du schéma incitatif quand il aboutit à des effets contraires à ceux recherchés.

Les incitations extrinsèques entrent en conflit avec les motivations intrinsèques quand l'agent mobilise la politique du principal pour découvrir la façon dont il le perçoit. Une politique incitative présente des « coûts cachés » en révélant à l'agent une information sur la confiance accordée par le principal. Elle modifie la représentation de l'interaction du côté de l'agent. L'intérêt d'un telle approche est de mettre l'accent sur la façon dont les individus comprennent les mécanismes incitatifs qui leurs sont destinés. Elle accorde une grande importance à la capacité d'interprétation des agents (Da Silva, 2011).

Cependant, si l'effet d'éviction est possible, il n'est pas certain car toutes les incitations financières ne sont pas contreproductives. Il convient alors d'identifier les conditions d'un possible effet d'éviction. Le terrain médical est un candidat crédible à une telle hypothèse. En effet, la spécificité de la profession réside dans son éthos fondé sur le care et l'obligation et qui dépasse les intérêts pécuniaires. Soigner voire guérir sont des motivations qui peuvent se suffire à elles mêmes. Les valeurs hippocratiques constituent les valeurs primaires de la profession médicale et le socle minimal de l'activité du médecin (Jaunait, 2005). Cette bienveillance médicale historique n'a pas besoin de stimuli financiers.

Dans ce contexte, assigner une valeur monétaire au temps de travail du médecin modifie la culture de la médecine. «Caregivers should be appropriately reimbursed but should not be constantly primed by money. Success in such a model will require collegiality, cooperation and teamwork - precisely the behaviors that are predictably eroded by a marketplace environment » (Hartzband et Groopman, 2009, p 102). Cette thématique a fait l'objet récemment de plusieurs tests empiriques sur les professionnels de santé (Gené-Badia et al. (2007), McDonald et al. (2007), Frolich et al. (2007)). L'examen de cette littérature révèle deux grands types d'enseignements.

(1) La conditionnalité des incitations. En mobilisant des données de panel concernant les mobiles de l'action de prévention de 525 médecins généralistes de la région PACA, Videau et al., (2009) ont cherché à identifier le type de médecins sensibles aux motivations intrinsèques. L'étude utilise une enquête de l'INSERM qui questionne les médecins selon la fréquence de leurs actions de prévention (proposer un sevrage tabagique ou une

\footnotetext{
${ }^{12}$ Voir Narcy (2007) pour une revue des expériences réalisées à partir de cette distinction.
} 
mammographie, calculer l'indice de masse corporelle). Elle cherche ensuite à connaître les médecins particulièrement sensibles à la prévention (le dernier quartile) en fonction de certaines variables que l'on peut associer à des MI (suivre des formations en santé publique, déclarer ne pas avoir besoin de rémunération spécifique, faire un don de temps) ou des ME (ne pas réaliser d'actes gratuits ou demander une aide en personnel car la prévention prend du temps). L'analyse vise ensuite à mesurer le poids relatif des MI par rapport au ME chez les médecins actifs en prévention. Elle montre que la part des MI est plus importante pour les médecins ne pratiquant pas de dépassement d'honoraires et qu'elle suit une courbe en $\mathrm{U}$ en fonction de l'âge : les MI sont plus prégnantes en début et en fin de carrière. Ce travail ne permet pas de conclure à l'existence d'un effet d'éviction. Il montre néanmoins que l'efficacité d'un contrat incitatif n'est pas garanti dans le domaine de la prévention alors même qu'il se développe en France actuellement avec le contrat d'amélioration des pratiques individuelles (CAPI). D'autres études empiriques (Ventelou et Rolland, 2009, Videau, 2010, Ventelou et Videau, 2011) soulignent l'hétérogénéité des motivations professionnelles et la variabilité des comportements. Les incitations financières ne sont pas efficaces partout et pour tous en médecine.

(2) Une autre littérature insiste sur le risque que font peser les incitations monétaires sur le sentiment d'autonomie au travail. Quand l'agent attribue sa performance à l'environnement plus qu'à son propre effort, une incitation forte signale la difficulté de la tache à réaliser et indique un fort doute du principal en la capacité de l'agent à réaliser cette tache. Cette configuration de « locus-of-control » externe ${ }^{13}$ accélère l'effet d'éviction. Le développement de la rémunération à la performance en médecine de ville comme à l'hôpital selon les recommandations des rapports Larcher et Aboud est de nature conduire à un phénomène de «sur justification ». De telles récompenses financières peuvent en effet être interprétées comme des injonctions malencontreuses par les personnels soignants invités à admettre qu'il faut les payer plus pour faire leur travail. Dans ce cas de figure, la récompense financière change la signification de l'acte qui n'est plus justifiée en elle même mais par la stimulation financière. En conséquence, elle peut être considérée comme une atteinte au sentiment de maîtrise de son activité et au besoin d'autonomie dans le travail et, in fine, détériorer les motivations intrinsèques. En érodant la capacité à influencer, par son comportement interne, le cours des choses, la récompense financière peut peser négativement sur l'estime de soi. L'enquête de Forest (2008) auprès d'un échantillon de 2502 médecins exerçant au sein d'établissements publics de santé a cherché à isoler la notion de besoin d'autonomie au travail. L'étude montre l'existence d'un niveau élevé de motivation autonome chez les médecins enquêtés. Or, l'effet potentiellement contreproductif des incitations monétaires est lié au degré de motivation autonome. L'introduction d'une rémunération à la performance peut alors être perçue comme un outil à caractère contrôlant (et conditionné à l'atteinte d'objectif fixé) et, à ce titre, compromettre l'objectif recherché. Ce constat rejoint la prudence affichée par le rapport de Bras et Duhamel (2008) sur le lien entre paiement à la performance et qualité des soins. L'étude des expériences de rémunération à la performance conduites au Royaume-Uni et aux Etats-Unis révèlent la difficulté de traduite en indicateurs mesurables ce qui relève du cœur du métier (poser un

\footnotetext{
${ }^{13}$ Dans une configuration de « locus-of-control » interne, l'agent croit que sa propre action détermine la récompense.
} 
bon diagnostic par exemple). Le degré d'acceptation du dispositif incitatif pèse aussi sur son efficacité. Ainsi, quand l'objectif d'une récompense financière est l'économie sur le coût des soins, le dispositif incitatif est moins bien accepté. De même, quand la nouvelle gouvernance hospitalière cherche les gains de productivité, qui incite à la disparition des temps gratuits source de lien social avec le patient, ou vise la promotion d'une culture de résultat qui oblige à rendre transparent ce qui ne peut l'être totalement, elle peut être vécue comme une atteinte à ce qui fait la fierté des soignants et le cœur de leur métier (Schweyer, 2010) et conduire à des phénomènes d'épuisement professionnel, intimement lié à l'absence de perte d'autonomie dans le travail.

Nous venons de relever l'affaiblissement des motivations intrinsèques associé aux dispositifs incitatifs portés par les réformes du système de santé. Nous allons maintenant examiner les effets de ces réformes sur l'équilibre entre valeurs hippocratiques et libérales ainsi que leurs conséquences paradoxales.

La recherche de l'adhésion des médecins à certains programmes de réduction des dépenses par des incitations tarifaires fait du prix l'élément principal de l'échange médical. La mise en avant de ces logiques de prix renforce les valeurs libérales au détriment des valeurs hippocratiques. En concentrant ainsi l'attention sur la seule dimension monétaire, cette politique change la conception qu'ont les médecins du « juste prix $»^{14}$. Elle suscite alors une augmentation des dépenses, à l'opposé de l'objectif visé. Nous parlons d'effets pervers des politiques de santé dans la mesure où celles-ci conduisent à augmenter les dépenses que l'on cherche à réduire. C'est le cas tout particulièrement de la nouvelle donne tarifaire créée par le secteur 2 dit à honoraires libres. Celle-ci est justifiée par le législateur au nom de la réduction des dépenses d'assurance maladie. Le dispositif vise à lutter contre la nature inflationniste du paiement à l'acte et à éviter que les médecins multiplient les actes pour garantir leurs revenus. L'augmentation du prix est vue comme un moyen de compenser la perte de revenu, induite par la décroissance recherchée du nombre d'actes. Elle doit inciter les médecins à réduire les quantités. L'instauration de ce secteur s'inscrit également dans contexte institutionnel français où la négociation tarifaire est un terrain d'expression principal du bras de fer récurent entre profession et Etat

L'instauration de ce secteur 2 semble avoir modifié les représentations de l'ensemble des médecins dans un sens davantage marchand. Son gel en $1990^{15}$ et l'encadrement des dépassements d'honoraires fait qu'il concerne de moins en moins de médecins généralistes. Pour autant, les dépassements d'honoraire ne peuvent être circonscrits au secteur 2. L'instauration du double secteur a installé une pratique du dépassement d'honoraires durable qui est aussi «adoptée » par les médecins du secteur 1 . Tout se passe comme si l'extinction programmée du secteur 2 achoppait sur une diffusion des dépassements d'honoraires audelà de ce secteur. Elle a conduit à modifier également les comportements des médecins restés au tarif conventionnel (Leroy, 2008).

Nous avons cherché à étayer cette hypothèse par une enquête visant à recueillir les justifications des médecins sur leurs pratiques tarifaires et à travers elles l'image de la bonne

\footnotetext{
${ }^{14}$ Celui que doit fixer le médecin en interprétant le « tact et mesure » du code de déontologie et le tarif opposable des conventions médicales, en fonction de caractéristiques diverses comme le revenu supposé du patient, son niveau de couverture complémentaire, le type d'acte ou sa durée, etc.

${ }^{15}$ Date à partir de laquelle les médecins déjà en secteur 2 qui le souhaitaient y sont demeurés, tandis que les autres ne pouvaient plus y accéder (à l'exception des anciens chefs de clinique-assistants).
} 
pratique médicale que se fait chaque médecin (Bloy et al., 2008) ${ }^{16}$. La banalisation et la contagion des dépassements d'honoraires dont témoigne cette enquête montre que des comportements de type secteur 2 se sont diffusés aux médecins du secteur 1 et non l'inverse. La pratique de dépassement influe sur les représentations de l'ensemble des professionnels. Ceci confirme que la création du secteur 2 et sa gestion ont affecté non seulement les médecins en secteur 2, comme on pouvait s'y attendre, mais aussi les médecins qui ne sont pas dans ce secteur tarifaire. La banalisation du dépassement d'honoraires existe aussi pour ceux qui ont volontairement opté pour le secteur 1 ! La création du secteur 2 développe, au delà de ce seul secteur, une conception de l'activité médicale où les actes chers sont jugés légitimes. On a ici une illustration de l'influence que peut avoir cette mesure de marchandisation de l'acte médical sur les représentations de l'ensemble des médecins.

Ces effets pernicieux et de longue portée de la création du double secteur se manifestent également dans la multiplication des dépassements sauvages, en ambulatoire comme à l'hôpital. Ces nouvelles pratiques tarifaires particulièrement saillantes au début des années 2000 semblaient totalement acceptables pour certains médecins au point qu'elles ont fait l'objet d'un recours en justice. Ainsi en est-il de la plainte déposée par une dermatologue, installée depuis mars 2000 et qui demande en 2002 un droit à dépassement alors que le secteur 2 n'a été ouvert que de 1980 à 1990. Le tribunal des affaires de Sécurité sociale de Boulogne-sur-Mer lui donne raison et la cour d'appel de Douai confirme le jugement à la suite de l'appel interjeté par la CPAM ${ }^{17}$. De même, en 2004, 57 médecins du Loiret ont donné une large publicité à leur auto proclamation en honoraires libres alors qu'ils ont été assignés par la CPAM d'Orléans devant le tribunal administratif. Ce combat pour une « juste rémunération » conduit à l'envolée des dépassements, y compris en secteur 1 comme le confirment les études statistiques de la $\mathrm{CNAM}^{18}$, ce qui offre un témoignage particulièrement saisissant de l'évolution de ce qu'il apparaît correct de faire suite à la permissivité marchande.

Ce constat rejoint celui de l'IGAS (Aballea et al., 2007) qui a publié un rapport alarmant sur ces pratiques tarifaires, en montrant que la fermeture du secteur 2 a pu enrayer le développement des dépassements mais n'a pas conduit à les éteindre. Au contraire, le taux de dépassement a doublé entre 1990 (année de gel du secteur 2) et 2002. L'importance des dépassements, leur concentration géographique, le monopole du secteur 2 pour certaines spécialités médicales remettent en question le principe d'égal accès aux soins.

En dépit des tentatives erratiques de geler l'accès au secteur à honoraires libres, il demeure important pour la médecine de spécialité et constitue aujourd'hui l'une des principales sources des inégalités d'accès aux soins avec une hausse des dépassements de $68 \%$ en euros constants entre 1990 et 2006. Certains départements n'ont aucun médecin spécialiste à tarif administré dans certains domaines (par exemple urologie ou chirurgie). Le montant du dépassement peut être très lourd : en moyenne 225 euros en hôpital public

\footnotetext{
${ }^{16}$ Voir aussi l'article de Abecassis et Domin dans ce numéro

${ }^{17}$ La raison donnée est que tout médecin doit avoir reçu par courrier le règlement conventionnel minimal de 1998, suite à la dénonciation de la convention. Or, la CPAM ne peut pas faire la preuve de l'envoi de ce règlement à la plaignante. Dans ce cas, le règlement ne s'applique pas. Voir Prétot (2004) pour un développement.

${ }^{18}$ Le nombre de consultations donnant lieu à un dépassement d'honoraires a été multiplié par deux en 2002 et les dépassements ont majoré les honoraires de $8 \%$ en moyenne en 2002 (CNAMTS, 2003).
} 
et 454 euros en clinique privée pour une prothèse totale de hanche. La création d'un vaste espace de liberté tarifaire consacre la déconnection entre les tarifs de la Sécurité sociale et ceux réellement pratiqués par les médecins. «Cette extension non maîtrisée des dépassements n'a guère à voir avec la recherche d'un recours plus pertinent aux soins, dans un contexte où l'information des patients est de surcroît loin d'être transparente. Elle conduit à de fortes disparités du coût effectif des soins, à une application de moins en moins générale des principes de solidarité liés à l'assurance maladie obligatoire, et à la raréfaction sur certains territoires d'une offre de soins financièrement accessible » (Elbaum, 2008, p. 4). L'érosion du tarif opposable, en fragilisant l'accès aux soins ébranle l'un des fondements de l'assurance maladie et du même coup l'édifice de 1945 (Tabuteau, 2003). Le recul du tarif opposable produit mécaniquement une hausse du reste à charge pour l'assuré ou sa complémentaire. «On assiste finalement à un renversement de la logique conventionnelle. Tandis que les tarifs d'honoraires sont de moins en moins opposables aux médecins, puisque ceux-ci revendiquent la possibilité de pratiquer des dépassements, à l'inverse, les dépassements tarifaires sont de plus en plus opposables aux assurés » (Vacarie et al., 2008, p. 1114).

La situation est particulièrement défavorable pour les plus démunis quand elle conduit à un refus de soins. Ainsi, la création de la CMUC, qui impose des actes gratuits ${ }^{19}$ pour certains patients, a engendré des effets pervers parce qu'elle s'est heurtée à la diffusion et à la banalisation des dépassements d'honoraires qui est la première cause du refus de soins (Despres, 2005 ; Despres et Naiditch, 2006, Carle, 2007, Ghirardello et Joly, 2007, Kerleau et Outin, 2011). Ce refus de soins est ainsi le résultat de la permissivité tarifaire, rendue réalisable par le nouveau contexte normatif induit par le droit et la politique publique.

Dans le même temps, la contagion et la banalisation des actes chers au-delà du secteur 2 se conjuguent avec l'érosion des actes gratuits. L'analyse statistique sur un panel de 8002 médecins libéraux observés durant la période allant de 1979 à 2000 montre l'existence d'une érosion des actes gratuits avec le temps. Cette érosion, dans un contexte où l'activité médicale s'accroît, n'est pas linéaire : un premier décrochage se produit en 1980 qui coïncide avec l'ouverture du secteur 2 et l'autre en 1993 (Lievaut et al., 2008). L'analyse révèle l'existence d'une corrélation entre les actes gratuits et les actes payants. Une telle corrélation était jusqu'à présent imputée à des effets de demande : les actes gratuits sont associés à une forme extrême d'un altruisme médical où la discrimination par les prix témoigne d'un « caring médical » sélectif selon que le patient est un proche, un confrère, un cas clinique saillant ou encore un patient sans ressource (Kronick, 2001). L'analyse économétrique montre au contraire que l'érosion des actes gratuits est, pour une part, indépendante des effets dus à la demande de soins (Lievaut, 2010, Lievaut et Batifoulier, 2011). Elle est à relier à un effet de marchandisation selon lequel le médecin est conduit à faire payer ce qu'il effectuait gratuitement naguère (Batifoulier et Ventelou, 2008). La transformation d'actes gratuits en actes payants et l'anomie d'une préférence altruiste du médecin conditionné à un type particulier de patients ou de soins sont une conséquence d'un environnement de politique économique, dominé par les considérations financières. L'opportunisme, dont font preuve les médecins en tarifant les actes gratuits, peut alors être

19 L'acte est gratuit pour le patient mais fait l'objet d'une rémunération pour le médecin au tarif de la Sécurité sociale. Il s'agit donc d'une gratuité différente de celle de l'acte non lucratif où le médecin refuse de percevoir des honoraires. 
une réaction à ce contexte de politique économique, qui constitue une information envoyée par la tutelle et interprétée par le médecin. La politique économique mise en œuvre peut alors présenter des « coûts cachés » en modifiant la représentation de l'interaction du côté du médecin d'un comportement conforme ou adéquat.

\section{Conclusion}

Ce travail a cherché à situer la marchandisation initiée par la puissance publique sur un plan normatif. Il est acquis que le marché de la science économique comme le modèle d'individu qui lui correspond (l'homo reconomicus) sont des fictions théoriques. La critique du réalisme des hypothèses est jugée banale, facile et finalement artificielle tant la théorie vise un modèle idéal. C'est sur ce plan normatif que l'analyse a été menée pour montrer que la fiction théorique vient à « performer» la réalité (Callon, 1998) quand sont activés, par les incitations, le calcul coût-avantage et la recherche de l'intérêt lucratif. La fiction n'en est plus totalement une quand elle vise à montrer que les comportements calculateurs que requiert le marché sont des hypothèses non seulement plausibles mais aussi probables, y compris dans le secteur de la santé. La coordination marchande peut alors constituer, même sous forme d'approximation, un bon modèle de la réalité. L'institution d'un marché en santé requiert la construction du modèle d'individu qui lui correspond. C'est pourquoi, la figure de l'homo æeconomicus est le fer de lance de cette marchandisation. En cherchant à se désencastrer de principes éthiques et de valeurs autres que l'intérêt instrumental, la politique de santé diffuse une normativité marchande.

Les caractéristiques intrinsèques du bien santé (bien collectif, externalités, etc.) l'éloignent d'un traitement par le marché. Il est alors au total plus facile de construire un marché de la santé en recherchant des comportements marchands que des biens marchands. L'activation de l'intéressement individuel dans le secteur de la santé permet de tracer cette trajectoire marchande. Puisqu'elle est supportée par des représentions normatives, cette orientation marchande modifie le sens que les agents donnent au système de santé. Elle transforme l'éthique médicale. En activant la dimension libérale de la médecine tout en minant sa dimension hippocratique, la politique publique a modifié les représentions d'une activité médicale légitime dont on trouve trace dans des comportements tarifaires nouveaux. Elle n'est alors pas sans danger au regard même des objectifs poursuivis par la puissance publique. Elle n'est pas exempte d'effets pervers et paradoxaux en activant des dépenses ou en accentuant les problèmes d'iniquité dans l'accès aux soins. 


\section{Bibliographique}

ABALLEA P., BARTOLI F., ESLOUS L. et YENI I., (2007), Les dépassements d'honoraires médicaux, Rapport IGAS n RM 2007-054P, Paris.

ABECASSIS P. et DOMIN J.-P., (2009), L'évolution des conventions médicales à travers leur discours. Les maux de la politique économique de santé in Barnay T., Legendre F. (Eds), Emploi et politiques sociales. Tome 1 : défis et avenirs de la protection sociale., Éditions de L’Harmattan, Paris, 71-84.

BATIFOULIER P. (ed), (2001), Théorie des conventions, Economica, Paris.

BATIFOULIER P., DOMIN J.P. et GADREAU M., (2008), Mutation du patient et construction d'un marché de la santé, Revue française de socio-économie 1,27-46.

BATIFOULIER P., EYMARD-DUVERNAY F et FAVEREAU O., (2007), État social et assurance maladie. Une approche par l'économie des conventions », Économie Appliquée 1, 203-229.

BATIFOULIER P. et GADREAU M., (2006), Régulation et coordination du système de santé. Des institutions invisibles à la politique économique in Eymard-Duvernay F. (ed), L'économie des conventions. Approfondissements, La Découverte, Paris, 453-468.

BATIFOULIER P. et VENTELOU B., (2008), L'érosion de la part gratuite en médecine libérale in Chanial P. (ed), La société vue du don, La Découverte, Paris, 443-460.

BECKER H.S., (2001), Les ficelles du métier, La Découverte, Paris.

BENABOU R. et TIROLE J., (2003), Intrinsic and extrinsic motivation, Review of economic studies 70 (3), 489-520.

BESSIS F., CHASERANT C., FAVEREAU O. et THEVENON O., (2006), L'identité sociale de l'homo conventionalis in Eymard-Duvernay F. (ed.), L'économie des conventions. Méthodes et résultats, tome 1, Débats, La Découverte, Paris,181-195.

BESSIS F. et FAVEREAU O., (2011), Le marché contre les professions in BATIFOULIER P., BUTTARD A. et DOMIN J.-P., Santé et politiques sociales : entre efficacité et justice. Autour des travaux de Maryse Gadreau, Editions Eska, Paris, 82-92.

BLOY G., BIENCOURT O., BATIFOULIER P., BILON I et LIEVAUT J., (2008), L'inscription tarifaire des médecins et la marchandisation par les prix. Une approche empirique à partir d'une enquête spécifique in P. Batifoulier, M. Gadreau et I. Vacarie (eds), «La gouvernance de l'assurance maladie : l'orientation marchande et ses paradoxes », Rapport final pour la Mire-DREES, Paris, 135-166.

BOLTANSKI L., THEVENOT L., (1991), De la justification. Les économies de la grandeur, Gallimard, Paris.

BRAS P.L. et DUHAMEL G., (2008), Rémunérer les médecins selon leurs performances : les enseignements des expériences étrangères, Inspection générale des affaires sociales, Paris.

BRUNO I., (2008), A vos marques, prêts... cherchez !, La stratégie européenne de Lisbonne, vers un marché de la recherche, Éditions du Croquant, Paris.

CALLON M., (ed), (1998), The laws of the Markets, Blackwell Publishers, Oxford.

CHAMPY F., (2009), Sociologie des professions, PUF, Paris.

CHAMPY F., (2011), Nouvelle sociologique des professions, PUF, Paris.

CHANIAL P., (2010), Le New Public Management est-il bon pour la santé ? Bref plaidoyer pour l'inestimable dans la relation de soin, Revue du MAUSS 35, 135-150

CNAMTS, (2003), Les dépassements d'honoraires médicaux entre 2000 et 2002, Point de conjoncture 11, 18-26.

DA SILVA, (2011), Actes gratuits et comportement du médecin. Une étude empirique, Communication au séminaire d'économie politique de la santé, Paris

DECI E.L., (1971), Effect of externally mediated rewards on intrinsic motivation, Journal of Personality and Social Psychology 18, 105-115.

DESPRES C. et NAIDITCH M., (2006), Analyse des attitudes de médecins et de dentistes à l'égard des patients bénéficiant de la Couverture maladie universelle. Une étude par testing dans six villes du Val-de-Marne, Ministère de la Santé-Fonds CMU, Paris. 
DESPRES C., (2005), La couverture maladie universelle : des usages sociaux différenciés, Sciences Sociales et Santé 23 (4), 79-108.

DIAZ-BONE R. et THEVENOT L., (2010), « La sociologie des conventions. La théorie des conventions, élément central des nouvelles sciences sociales françaises », Trivium, 5-2010, [En ligne], URL : http:// trivium.revues.org/index3626.html.

DOMIN J-P., (2008), Une histoire économique de l'hôpital, Tome 1, Comité d'histoire de la sécurité sociale, La Documentation française, Paris.

DOMIN J.-P., (2010), La nouvelle gouvernance de l'assurance maladie : la consécration d'une régulation marchande ?, Économie et Institutions 15, 5-29.

ELBAUM M., (2008), Participation financière des patients et équilibre de l'assurance maladie, Lettre de l'OFCE 301.

EYMARD-DUVERNAY F., (1989), Convention de qualité et formes de coordination, Revue Économique 40 (2), 329-359.

EYMARD-DUVERNAY F., (2004), Économie politique de l'entreprise, La Découverte, Paris.

EYMARD-DUVERNAY F. (ed), (2006), L'économie des conventions, méthodes et résultats, tome 1 : Débats, tome 2 : Développements, La Découverte, Paris.

EYMARD-DUVERNAY F., (2009), L'économie des conventions entre économie et sociologie : l'homo conventionalis calcule et parle in Steiner P., Vatin F. (eds), Traité de sociologie économique, PUF, Paris, 131-164.

FAVEREAU O., (1988), La Théorie Générale : de l'économie conventionnelle à l'économie des conventions, Cahier d'économie politique 14-15, L'Harmattan, Paris, 197-220.

FAVEREAU O., (1989), Marchés internes, marchés externes, Revue économique 40 (2), 273-328.

FAVEREAU O., (2010), Les avocats entre ordre professionnel et ordre marchand, Lextenso, Paris.

FAVEREAU O., (2010b), La place du marché in A. Hatchuel, O. Favereau et F Aggeri (eds) : L'activité marchande sans le marché ? Colloque de Cerisy, Presses des mines, Paris, 111-131.

FAVEREAU O., BIENCOURT O., EYMARD-DUVERNAY F. (2002), Where do market come from ? From (quality) conventions ! in Favereau O., Lazega E. (eds), Conventions and Structures in Economic Organization: Markets, Networks, and Hierarchies, Edward Elgar Publishing, Cheltenham, 213-252.

FOREST V., (2008), Nouvelle Gestion Publique et comportements au travail : l'exemple des médecins de la Fonction Publique Hospitalière, Thèse pour le doctorat de sciences de gestion, Université Lumière Lyon 2.

FREY B. S., (1997), Not just for the money : an economic theory of personal motivation, Edward Elgar, Cheltenham.

GADREAU M., (2009), Économie et éthique en santé. Du calcul économique aux jugements de valeurs, Revue de Philosophie Economique 10 (1), 3-17.

GADREAU M. et VIDEAU Y., (2008), Un paradoxe de gouvernance pour l'assurance maladie ?, Journées gouvernance, Laboratoire d'Économie et de Gestion, CNRS-Université de Bourgogne, Dijon, 29-30 Mai.

GHIRARDELLO A. et JOLY L., (2007), État de santé, accès aux soins et discrimination : quelles perspectives pour 1'Etat social ?, Économie Appliquée 1, 231-256.

GILLON R., (1994), Medical ethics: four principles plus attention to scope, British Medical Journal 309, 184190.

GNEEZY U. et RUSTICHINI A., (2000), Pay enough or don't pay at all, Quarterly Journal of Economics 115, 791-810

GRIGNON M., (2006), La question de la régulation des systèmes de santé. Eléments d'analyse économique, Revue Française des affaires sociales, Réforme et régulation des systèmes de santé en Europe, 2-3, avrilseptembre, 43-62.

HASSENTEUFEL P., (1997), Les médecins face à l'État, Presses de sciences politiques, Paris. 
HATZFELD H., (1963), Le grand tournant de la médecine libérale, Éditions ouvrières, Paris.

HARTZBAND P. et GROOPMAN J., (2009), Money and the Changing Culture of Medicine, New England Journal of Medicine 360, 101-103.

HIRSCHMAN A.O., (1985), Trois façons simples de compliquer el discours de l'économie politique in Le sens commun. Vers une économie politique élargie. Les éditions de Minuit, Paris.

HIRSCHMAN A.O., (1991), Deux siècles de rhétorique réactionnaire, Fayard, Paris.

JAUNAIT A., (2005), Comment pense l'institution médicale? Une analyse des codes français de déontologie médicale, Éditions Dalloz, Paris.

KARPIK L., (2007), L'économie des singularités, Gallimard, Paris.

KERLEAU M. et OUTIN J-L., (2011), Pauvreté et CMU : fragilité des populations, fragilité des droits in Batifoulier P., Buttard A., Domin JP. (eds) Santé et politiques sociales : entre efficacité et justice. Autour des travaux de M. Gadreau, ESKA, Paris, 171-182.

KREPS D. M., (1997), Intrinsic motivation and extrinsic incentives, American economic review 87 (2), 353-364.

KRONICK R., (2001), Valuing charity, Journal of Health Politics, Policy and Law 26 (5), 993-1001.

LAUDE A, (2005), Les médecins et les malades face au droit, Revue française d'administration publique 113, 113-122.

LEMIEUX C., (2009), Le devoir et la grâce, Economica, Paris.

LEMIEUX C. (ed), (2010), La subjectivité journalistique. Onze leçon sur le rôle de l'individualité dans la production de l'information, éditions de l'EHESS, Paris.

LEMIEUX C., (2010b), Un président élu par les médias ? Regard sociologique sur la présidentielle de 2007, Presses des Mines, Paris.

LEROY S., (2008), Les faiblesses du mécanisme conventionnel in P. Batifoulier, M. Gadreau et I. Vacarie (eds.), La gouvernance de l'assurance maladie : l'orientation marchande et ses paradoxes, Rapport final pour la Mire-DREES, 24-30.

LIEVAUT J., (2010), Le « maillon faible » de la régulation des dépenses de santé en France : Les comportements inattendus des médecins libéraux. Thèse de Doctorat de Science Économique, Université Paris Ouest Nanterre La Défense, Nanterre.

LIEVAUT J., BATIFOULIER P. et GANNON F., (2008), Actes gratuits et comportement du médecin. Une étude empirique in Domin J.-P., Maric M., Delabruyere S., Hédouin C., (eds) Actualité de l'économie sociale, L'Harmattan, Paris, 193-205.

LIEVAUT J. et BATIFOULIER P., (2011), La transformation des actes gratuits en actes payants en médecine libérale. Une étude micro-économétrique longitudinale, Document de Travail EconomiX 2011-12.

McDONALD R., HARRISON S., CHECKLAND K., CAMPBELL S. et ROLAND M., (2007), Impact of financial incentives on clinical autonomy and internal motivation in primary care: ethnographic study, British Medical Journal 334, 1357-59.

MOISDON J-C., (2000), Quel est la valeur de ton point ISA ? Nouveaux outils de gestion et de régulation dans le système hospitalier français, Sociologie du travail (42), 31-49.

MOSSE P. et PIERRU F., (2002), Entre savoirs économiques et décisions politiques : les restructurations hospitalières in Béjean S., Peyron S., Santé, Règles et Rationalité, Economica, Paris, 235-268.

MOSSE P., (2005), Vers la construction d'un nouvel espace professionnel médical in Batifoulier P. et Gadreau M., (eds), Éthique médicale et politique de santé, Economica, Paris, 123-141.

NARCY M., (2007), Motivation intrinsèque et équité salariale. Une comparaison ente le secteur associatif et le secteur privé, Thèse de doctorat de sciences économiques, Paris II.

NYE R., (2006), Médecins, éthique médicale et Etat en France 1789-1947, Le mouvement social 214, 19-36.

ORLEAN A., (2004), L'économie des conventions : définitions et résultats, in Orléan A. (ed.), Analyse économique des conventions, PUF, Paris, 9-49. 
PRETOT X., (2004), Le contentieux du changement de secteur tarifaire des médecins spécialistes, Droit social 9/10,877-882.

REVUE ÉCONOMIQUE, (1989), numéro spécial « économie des conventions », 40 (2).

ROBELET M., (2005), Réformes du système de santé et mobilisation éthique des médecins : un regard sociologique sur l'éthique des professions in Batifoulier P. et Gadreau M. (eds.), Ethique médicale et politique de santé, Economica, Paris, p. 145-166.

ROCHAIX L., (2004), Concurrence et assurance santé, entre vœux pieux et vieux démons, Revue d'Économie Politique 114 (5), 575-576.

SALAIS R. et THEVENOT L. (eds), (1986), Le Travail : marchés, règles, conventions, INSEE, Economica, Paris.

SALTMAN R., (2002), Regulating incentives: the past and present role of the state in health care systems, Social Science and Medecine, 54 (11), 1677-1694.

SAVAGE L.J., (1954), The Foundations of Statistics, Dover Publication, New York.

SALAIS R. et STORPER M., (1995), Les Mondes de production. Enquête sur l'identité économique de la France, éditions de l'EHESS, Paris.

SCHWEYER F-X. , (2010), Santé, contrat social et marché : la fonction publique hospitalière en réformes, Revue française d'administration publique 132, 727-744.

SHAKED A. et SUTTON J., (1981), The Self-Regulation Profession, Review of Economic Studies XLVIII, 217-234.

SVANDRA P., 2007, Le soin, une éthique au cour de la vie, Thèse pour le doctorat de philosophie, Université de Marne la Vallée.

TABUTEAU D., (2003), La liberté tarifaire ?, Droit social 4, 424-426.

THEVENOT L., (1985), Les investissements de forme, in Conventions économiques, Cahiers du CEE, Paris, PUF, 21-71.

THEVENOT L., (1992), Un pluralisme sans relativisme ? Théories et pratiques du sens de la justice, in Affichard, J., de Foucauld, J.-B. (éds.), Justice sociale et inégalités, Paris, Ed. Esprit, pp.221-253.

THEVENOT L., (2006), L'action au pluriel, Sociologie des régimes d'engagement, La Découverte, Paris.

TIROLE J., (2009), Motivation intrinsèque, incitations et normes sociales, Revue économique 60 (3), 577-589.

VACARIE I., ALLOUACHE A., GINON A-S., FERKANE Y. et LEROY S., (2008), Crise de l'État-providence ou crise de la régulation économique ? Les leçons des réformes de l'assurance maladie, Droit social, novembre 11,1103- 1114 .

VALAT B., (2001), Histoire de la sécurité sociale (1945 - 1967). L'Etat, l'institution et la santé, Economica, Paris.

VAUCHEZ A. et WILLEMEZ L. (dir), (2007), La justice face à ses réformateurs (1980-2006). Entreprises de modernisation et logiques de résistance, Paris, PUF.

VENTELOU B. et ROLLAND S., (2009), Evaluation de l'implication des médecins généralistes libéraux dans le bon usage des médicaments, Santé publique 21, 129-138.

VENTELOU B. et VIDEAU Y., 2011, De la motivation des médecins généralistes français, Santé et Politiques sociales: entre efficacité et justice/ed. par Ph. Batifoulier, A. Buttard et J.-P. Domin. ESKA, pp. 93-105.

VIDEAU Y., (2010), Essais sur l'offre de travail en médecine générale. Du rôle des incitations et des motivations. Thèse de Doctorat de Sciences Économiques, Université de la Méditerranée - Aix Marseille II.

VIDEAU Y., BATIFOULIER P., ARRIGHI Y., VENTELOU B. et GADREAU M., (2010), Le cycle de vie des motivations professionnelles en médecine générale : une étude dans le champ de la prévention, Revue d'Épidémiologie et de santé publique 58 (5), 301-311. 


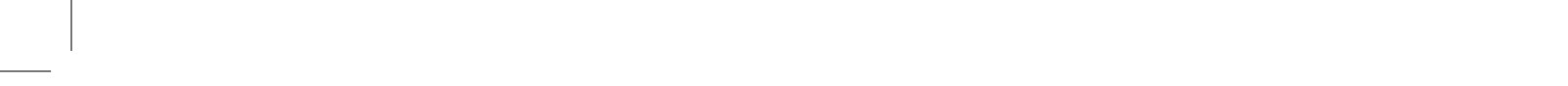

